\title{
Non oscillatory nonlinear differential systems with slowly varying coefficients in presence on certain damping forces
}

\author{
Pinakee Dey \\ Department of Mathematics, Mawlana Bhashani Science and Technology University, Santosh, Tangail-1902, Bangladesh
}

\section{Email address:}

pinakee_68@yahoo.com (P. Dey)

\section{To cite this article:}

Pinakee Dey. Non Oscillatory Nonlinear Differential Systems with Slowly Varying Coefficients in Presence on Certain Damping Forces. Pure and Applied Mathematics Journal. Vol. 2, No. 1, 2013, pp. 28-31. doi: 10.11648/j.pamj.20130201.14

\begin{abstract}
Krylov-Bogoliubov-Mitropolskii method is modified and applied to certain damped nonlinear systems with slowly varying coefficients. The results obtained by this method show excellent coincidence with those obtained by numerical method. The method is illustrated by an example.
\end{abstract}

Keywards: Perturbation Methods, Varying Coefficient, Unperturbed Equation, Nonlinear Differential Systems, Damped System

\section{Introduction}

The Krylov-Bogoliubov-Mitropolskii (KBM) method [1-3] is well known in the theory of nonlinear oscillations. The method was originally developed by Krylov and Bogoliubov [1] for obtaining the periodic solution of non linear systems with small nonlinearities. Then the method was amplified and justified by Bogoliubov and Mitropolskii [2]. Mitropolskii [3] has extended the method to nonlinear differential system with slowly varying coefficients. On the other hand, Popov [4] extended the method to nonlinear damped oscillatory systems with constant coefficients. Murty, Deekshatulu, and Krisna [5] investigated an over damped nonlinear system using Bogoliubov's method. Murty [6] presented a unified KBM method for solving second order nonlinear systems which cover the un-damped, damped and over-damped cases. Shamsul [7] has presented a unified method for solving an $n$-th order differential equation (autonomous) characterized by oscillatory, damped oscillatory and non-oscillatory processes. Hung and $\mathrm{Wu}$ [8] obtained an exact solution of a differential system in terms of Bessel's functions where the coefficients varying with time in an exponential order. Roy and Shamsul [9] found an asymptotic solution of a differential system in which the coefficient changes in an exponential order of slowly varying time. Bojadziev and Edwards [10] studied some damped oscillatory and purely non-oscillatory systems with slowly varying coefficients. Recently Pinakee et.al [11-12] has presented extended KBM method (by Popov [4]) for solving nonlinear problems in which the coefficients change slowly and periodically with time. In accordance to Pinakee et.al [11-12] observation, [10]'s solution is not useful for strong damping effects. The aim of this article is to find an approximate solution for strong damping effect with varying coefficients in where [10]'s solution is unable to give desired results.

\section{Materials and Method}

Let us consider the nonlinear differential system

$$
\ddot{\mathrm{x}}+2 \mathrm{k}(\tau) \dot{\mathrm{x}}+\omega^{2}(\tau) \mathrm{x}=-\varepsilon \mathrm{f}(\dot{\mathrm{x}}, \mathrm{x}, \tau), \quad \tau=\varepsilon t
$$

where the over-dots denote differentiation with respect to $\mathrm{t}, \varepsilon$ is a small parameter, $k=\mathrm{O}(\varepsilon), \mathrm{f}$ is a given nonlinear function and $\omega(\tau)$ is known as frequency. For $\varepsilon=0$ and $\tau=\tau_{0}=$ constant, $k\left(\tau_{0}\right) \pm i \omega\left(\tau_{0}\right)$ are two eigen values of the unperturbed equation of (1) and has the solution

$$
x(t, 0)=\alpha_{0} e^{-k\left(\tau_{0}\right) t} \cos \left(\omega_{0}\left(\tau_{0}\right) t+\varphi_{0}\right)
$$

where $\alpha_{0}$ and $\varphi_{0}$ are arbitrary constant and $\tau_{0}$ represents the value of $\tau$ when $\varepsilon=0$.

When $\varepsilon \neq 0$ we seek a solution in accordance with the KBM method, of the form 


$$
\mathrm{x}(\mathrm{t}, \varepsilon)=\alpha \cos \varphi+\varepsilon \mathrm{u}_{1}(\alpha, \varphi, \tau)+\varepsilon^{2}
$$

where $\alpha$ and $\phi$ satisfy the equations

$$
\begin{aligned}
& \dot{\alpha}=-k(\tau)+\varepsilon A_{1}(\alpha, \phi, \tau)+\varepsilon^{2} \ldots \\
& \dot{\phi}=\omega_{0}(\tau)+\varepsilon B_{2}(\alpha, \phi, \tau)+\varepsilon^{2} \ldots
\end{aligned}
$$

Confining attention to the first few term $1,2 \ldots \mathrm{m}$ in the series expansion of (3) and (4), we evaluate functions $u_{1}, A_{1}$ and $B_{1}$ such that $\dot{\alpha}$ and $\dot{\phi}$ appearing in (3) and (4) satisfy (1) with an accuracy of $\varepsilon^{m+1}$. In order to determine these unknown functions it was early assumed by Murty [6], Shamsul [7] that the functions $u_{1}$ exclude all fundamental terms, since these are included in the series expansion (3) at order $\varepsilon^{0}$.

Now differentiating (6) twice with respect to t, substituting for the derivatives $\ddot{x}, \dot{x}$ and $\mathrm{x}$ in (1), utilizing relation (4) and comparing the coefficients of $\mathcal{E}$, we obtain

$$
\begin{aligned}
& \omega_{0} \alpha \sin \varphi\left(\begin{array}{c}
\mathrm{k} \alpha \frac{\partial}{\partial \alpha} \mathrm{A}_{1}-\mathrm{kA}_{1} \\
-2 \omega_{0} \alpha \mathrm{B}_{1}
\end{array}\right) \cos \varphi \\
& -\sin \varphi\left(\begin{array}{c}
-\mathrm{k} \alpha{ }^{2} \frac{\partial}{\partial \alpha} \mathrm{B}_{1} \\
+2 \omega_{0} \mathrm{~A}_{1}
\end{array}\right)+ \\
& \left(-\mathrm{k} \alpha \frac{\partial}{\partial \alpha}+\frac{\partial}{\partial \varphi}\right)^{2} \mathrm{u}_{1}+2 \mathrm{k}\left(\begin{array}{l}
-\mathrm{k} \alpha \frac{\partial}{\partial \alpha} \\
+\frac{\partial}{\partial \varphi}+\omega^{2}
\end{array}\right) \mathrm{u}_{1} \\
& =-\mathrm{f}^{(0)}(\alpha, \varphi, \tau),
\end{aligned}
$$

where $f^{(0)}=f\left(x_{0}, \dot{x}_{0}\right)$ and $x_{0}=\alpha \cos \phi$

It is assumed that both $f^{(0)}$ can be expanded in Fourier series [7]

$$
f^{(0)}=\sum_{n=0}^{\infty} F_{n}(\alpha) \cos n \phi+G_{n}(\alpha) \sin n \phi,
$$

and

$$
\begin{aligned}
& \mathrm{u}_{1}(\alpha, \varphi)=\mathrm{U}_{0}(\alpha)+\sum_{\mathrm{n}=2}^{\infty} \mathrm{U}_{\mathrm{n}}(\alpha) \cos \mathrm{n} \varphi \\
& +\mathrm{V}_{\mathrm{n}}(\alpha) \sin \mathrm{n} \varphi
\end{aligned}
$$

Substituting the expression for $f^{(0)}$ and $u_{1}$ in (8), we obtain the following equations for $A_{1}, B_{1}$ and $u_{1}$ as

$$
\begin{aligned}
k^{\prime} \alpha- & k \alpha \frac{\partial A_{1}}{\partial \alpha}+k A_{1}-2 \omega_{0} \alpha B_{1}=-F_{1} \\
& -\omega_{0}^{\prime} \alpha-2 \omega_{0} A_{1}+k \alpha^{2} \frac{\partial B_{1}}{\partial \alpha}=-G_{1}
\end{aligned}
$$

$$
\begin{aligned}
& \left(\left(-k \alpha \frac{\partial}{\partial \alpha}+\frac{\partial}{\partial \varphi}+k\right)^{2}+\omega_{0}^{2}\right) u_{1} \\
& =-F_{0}-\sum_{n=2}^{\infty} F_{n}(\alpha) \cos n \varphi+G_{n}(\alpha) \sin n \varphi
\end{aligned}
$$

The particular solution of (8)-(10) gives three unknown functions $A_{1}, B_{1}$ and $u_{1}$ which complete the determination of the first order Bojadziev and Edwards [10] solution of (1). It is clear that both functions $A_{1}$ and $B_{1}$ is independent of phase variable $\phi$ and $u_{1}$ excludes all first harmonic terms. In accordance to [7] assumption $A_{1}, B_{1}$ and $u_{1}$ satisfy the following equations (instead of (8)-(10))

$$
\begin{aligned}
& k^{\prime} \alpha-k \alpha \frac{\partial A_{1}}{\partial \alpha}+k A_{1}-2 \omega_{0} \alpha B_{1}=-F_{1} \cos ^{2}(\phi-\omega t) \\
& -\omega_{0}^{\prime} \alpha-2 \omega_{0} A_{1}+k \alpha^{2} \frac{\partial B_{1}}{\partial \alpha}=-G_{1} \cos ^{2}(\phi-\omega t) \\
& \left(\left(-\mathrm{k} \alpha \frac{\partial}{\partial \alpha}+\frac{\partial}{\partial \varphi}+\mathrm{k}\right)^{2}+\omega_{0}^{2}\right) \mathrm{u}_{1} \\
& =-\mathrm{F}_{0}-\left(\mathrm{F}_{1} \cos \varphi+\mathrm{G}_{1} \sin \varphi\right) \sin ^{2}(\varphi-\omega \mathrm{t})- \\
& \sum_{\mathrm{n}=2}^{\infty} \mathrm{F}_{\mathrm{n}}(\alpha) \cos \mathrm{n} \varphi+\mathrm{G}_{\mathrm{n}}(\alpha) \sin \mathrm{n} \varphi
\end{aligned}
$$

The particular solution of (11)-(13) gives three unknown functions $A_{1}, B_{1}$ and $u_{1}$. Thus the determination of the first order solution is clear. In this case $A_{1}$ and $B_{1}$ depend on both $\alpha$ and $\phi$ and $u_{1}$ is not independent of first harmonic terms.

Example: We consider a second order nonlinear system with constant and slowly varying coefficient

$$
\ddot{x}+2 k(\tau) \dot{x}+\omega(\tau) x=-\varepsilon x^{3},
$$

Here $f=x^{3}, f^{(0)}=\alpha^{3} \cos ^{3} \alpha=\frac{1}{4} \alpha^{3}(3 \cos \phi+\cos 3 \phi)$; so that non-zero coefficients are $F_{1}=\frac{3}{4}, F_{3}=\frac{1}{4}$. Substituting the values pf $F_{1}$ and $F_{3}$ into (11)-(13) and solving them, we obtain

$$
\begin{aligned}
& \mathrm{A}_{1}=-\frac{\mathrm{k}^{\prime} \alpha}{2 \omega_{0}}+\frac{3 \mathrm{k} \alpha^{3} \cos ^{2} \varphi_{0}}{8 \omega^{2}}, \\
& \mathrm{~B}_{1}=\frac{-\omega_{0}^{\prime}}{2 \omega_{0}}+\frac{3 \omega_{0} \alpha^{2} \cos ^{2} \varphi_{0}}{8 \omega^{2}}
\end{aligned}
$$

and

and 


$$
\begin{gathered}
u_{1}=\frac{3 \alpha^{3}\left(-\cos \phi+\omega_{0} \sin \phi / k\right) \sin ^{2} \phi_{0}}{16 \omega^{2}}+ \\
\frac{\alpha^{3}\left(-\left(k^{2}-2 \omega_{0}^{2}\right) \cos 3 \phi+3 k \omega_{0} \sin 3 \phi\right.}{16 \omega^{2}\left(k^{2}+4 \omega_{0}^{2}\right)} \\
\mathrm{A}_{1}=-\frac{\mathrm{k}^{\prime} \alpha}{2 \omega_{0}}+\frac{3 \mathrm{k} \alpha^{3}}{8 \omega^{2}}, \\
\mathrm{~B}_{1}=\frac{-\omega_{0}^{\prime}}{2 \omega_{0}}+\frac{3 \omega_{0} \alpha^{2}}{8 \omega^{2}}
\end{gathered}
$$

and

$$
u_{1}=\frac{\alpha^{3}\left(-\left(k^{2}-2 \omega_{0}^{2}\right) \cos 3 \phi+3 k \omega_{0} \sin 3 \phi\right.}{16 \omega^{2}\left(k^{2}+4 \omega_{0}^{2}\right)}
$$

Now substituting the functional values of $A_{1}, B_{1}$ from (15) into (4), we obtain

$$
\begin{aligned}
& \dot{\alpha}=-k(\tau) \alpha-\frac{k^{\prime} \alpha}{2 \omega_{0}}+\frac{3 k \alpha^{3} \cos ^{2} \phi_{0}}{8 \omega^{2}}, \\
& \dot{\phi}=\omega(\tau)-\frac{\omega_{0}^{\prime}}{2 \omega_{0}}+\frac{3 \omega_{0} \alpha^{2} \cos ^{2} \phi_{0}}{8 \omega^{2}}
\end{aligned}
$$

Therefore, the first order solution of the equation (14) is

$$
x(t, \varepsilon)=\alpha \cos \phi+\varepsilon u_{1}
$$

where $\alpha$ and $\phi$ are the solution of the equation (19) $u_{1}$ is given by (16). Substituting the values of $A_{1}, B_{1}$ from (17) into (4) and solving them, [10] found the solution of (4) similar to (19). In this paper, we have used the Runge-Kutta (fourth order) method. Numerically, it is advantageous; a large step size can be used in the integration (see [13] for detail).

\section{Results and Discussions}

In order to test the accuracy of an approximate solution obtained by a certain perturbation method, one compares the approximate solution to the numerical solution (considered to be exact). With regard to such a comparison concerning the presented KBM method (by Popov [4]) of this article, we refer to the works of Murty [6] and Shamsul [7]. In our present paper, for different damping forces, we compare solution (20) and [10]'s solution.

First of all, for damping force $k(\tau)=0.5 e^{-\frac{-1}{2} \tau}$ and for $\varepsilon=.1, x$ is calculated by (20) with initial conditions $[x(0)=1.00000 \quad \dot{x}=-0.00000]$. Then $x(\varepsilon, t)$ has been computed by [10]'s solution. Finally the numerical solution has been obtained and percentage error has been calculated. All the results are shown in Table 1. From Table 1 it is seen that errors of the results obtained by solution (20) and [10]'s solution are less than $1 \%$ and on an average percentage errors of [10]'s solution are less than those computed by solution (20). Secondly, for damping force $k(\tau)=.707 e^{-\frac{1}{2} \tau}$ and for $\mathcal{E}=.1, x$ is calculated by (20) with initial conditions $[x(0)=1.0000(\dot{x}=-0.00000]$. The results are given in Table 2. Table 2 shows that percentage errors of solution (20) occur in an order of $1 \%$ (except at $(t=3$ and $t=8)$, while errors of [10]'s are many times greater than $1 \%$ and almost twice of those obtained by solution (20). If the damping force is increased and strong, solution (20) shows a good coincidence with the numerical solution. Contrary, errors of [10]'s solution increase.

\begin{tabular}{llllll}
\multicolumn{5}{c}{ Table 1 } \\
\hline$t$ & $x$ & $x_{n u}$ & $E^{0} \%$ & $x_{B E}$ & $E_{B E} \%$ \\
\hline 0.0 & 1.000000 & 1.00000 & 0.0000 & 1.000000 & 0.0000 \\
1.0 & 0.642193 & 0.641101 & 0.1703 & 0.641957 & 0.1335 \\
2.0 & 0.136156 & 0.134311 & 1.3744 & 0.134611 & 0.2241 \\
3.0 & -0.140639 & -0.141801 & -0.8195 & -0.142327 & 0.3709 \\
4.0 & -0.192790 & -0.192868 & -0.0404 & -0.193747 & 0.4558 \\
5.0 & -0.131988 & -0.131299 & 0.5248 & -0.132070 & 0.5872 \\
6.0 & -.050690 & -0.049836 & 1.7136 & -0.050253 & 0.8367 \\
8.0 & 0.033573 & 0.033807 & -0.6922 & 0.033981 & 0.5147 \\
10 & 0.027473 & 0.027256 & 0.7962 & 0.027513 & 0.9429 \\
\hline
\end{tabular}

Table 2

\begin{tabular}{llllll}
\hline$t$ & $x$ & $x_{n u}$ & $E \%$ & $x_{B E}$ & $E_{B E} \%$ \\
\hline 0.0 & 1.000000 & 1.000000 & 0.00000 & 1.000000 & 0.00000 \\
1.0 & 0.678614 & 0.677989 & 0.0922 & 0.684010 & 0.8881 \\
2.0 & 0.265293 & 0.263317 & 0.7504 & 0.268888 & 2.1157 \\
3.0 & 0.03068 & 0.031519 & 4.9145 & 0.034209 & 8.5345 \\
4.0 & -0.049626 & -0.050305 & -1.3498 & -0.049713 & -1.1768 \\
5.0 & -0.057106 & -0.057181 & -0.1311 & -0.057548 & 0.6418 \\
6.0 & -0.039592 & -0.039411 & 0.4593 & -0.039994 & 1.4793 \\
8.0 & -0.007227 & -0.007075 & 2.1484 & -0.007350 & 3.8869 \\
10 & 0.002924 & 0.002941 & -0.5780 & 0.002932 & -0.3060 \\
\hline
\end{tabular}

\section{Conclusion}

Based on the works of extended KBM method an approximate solution of a second order nonlinear deferential system with slowly varying coefficients has been found. In this paper solution (20) gives satisfactory results for the strong damping effect but Bojadzeiv and Edwards's [10] solution fail to give desire result.

\section{References}

[1] N.N, Krylov and N.N., Bogoliubov, Introduction to Nonlinear Mechanics. Princeton University Press, New Jersey, 
1947.

[2] N. N, Bogoliubov and Yu. Mitropolskii, Asymptotic Methods in the Theory of nonlinear Oscillations, Gordan and Breach, New York, 1961.

[3] Yu.,Mitropolskii, "Problems on Asymptotic Methods of Non-stationary Oscillations" (in Russian), Izdat, Nauka, Moscow, 1964.

[4] I. P. Popov, "A generalization of the Bogoliubov asymptotic method in the theory of nonlinear oscillations", Dokl.Akad. Nauk SSSR 111, 1956, 308-310 (in Russian).

[5] I. S. N. Murty, B. L. Deekshatulu and G. Krisna, "General asymptotic method of Krylov-Bogoliubov for over-damped nonlinear system", J. Frank Inst. 288 (1969), 49-46.

[6] I.S.N. Murty, "A Unified Krylov-Bogoliubov Method for Second Order Nonlinear Systems", Int. J. nonlinear Mech., 6, 1971, pp45-53.

[7] M.,Shamsul Alam, "Unified Krylov- Bogoliubov- Mitropolskii Method for Solving n-th Order Nonlinear System with Slowly Varying Coefficients", Journal of Sound and Vibration, 256, 2003, pp987-1002.

[8] Cheng Hung and Tai Tsun Wu, An Aging Spring, Studies in
Applied Mathematics, 49, 1970. pp183-185.

[9] K.C. Roy and M. Shamsul Alam, Effects of Higher Approximation of Krylov- Bogoliubov-Mitropolskii Solution and Matched Asymptotic Solution of a Differential System with Slowly Varying Coefficients and Damping Near to a Turning Point, Vietnam Journal of Mechanics, VAST, 26, 2004.,pp182-192.

[10] G.,Bojadziev, and J. Edwards, "On Some Asymptotic Methods for Non-oscillatory and Oscillatory Processes", Nonlinear Vibration Problems, 20, 1981, pp69-79.

[11] Pinakee Dey., Harun or Rashid, M. Abul Kalam Azad and M S Uddin, "Approximate Solution of Second Order Time Dependent Nonlinear Vibrating Systems with Slowly Varying Coefficients", Bull. Cal. Math. Soc, 103, (5), 2011. pp 371-38.

[12] Pinakee Dey, Md. Babul Hossain, Md. Musa Miah and Mohammad Mokaddes Ali,.” Approximate solutions of damped nonlinear vibrating system with varying coefficients under some conditions" Applied and Computational Mathematics. 1(2), 2012, pp 1-6.

[13] A. H. Nayfeh, Introduction to perturbation Techniques, J. Wiley, New York, 1981. 